\title{
Synthese des Geranyl-Nerolidols
}

\section{Doctoral Thesis}

\section{Author(s):}

Madinaveitia, Luis Castro

\section{Publication date:}

1945

\section{Permanent link:}

https://doi.org/10.3929/ethz-a-000098897

\section{Rights / license:}

In Copyright - Non-Commercial Use Permitted 


\section{SYNTHESE DES \\ GERANYL-NEROLIDOLS \\ Von der}

Eidgenössischen Technischen Hochschule

in Zür'ich

zur Erlangung

der Würde eines Doktors der technischen Wissenschaften

GENEHMIGTE

\section{PROMOTIONSARBEIT}

VORGELEGT VON

\section{LUIS CASTRO MADINAVEITIA}

DIPL. INGENIEUR-CHEMIKER

AUS MADRID (SPANIEN)

REFERENT : HERK PROF, DR. L. RUZICKA

KORREFERENT : HERR PROF. DR. PL. A. PLATTNER

LAUSANNE

IMP RIMERIE T. GEN EUX

1945 


\section{Zusammenfassung.}

In der vorliegenden Arbeit wurde das bis jetzt unbekannte Geranyl-nerolidol synthetisch dargestellt. Die Verbindung, die nur mühsam erhalten werden konnte, war ein stark luftempfindliches Oel. Weitere Versuche zur Gewinnung des Farnesyl-farnesols sind deshalb sehr erschwert.

Es wurden die Bedingungen der Anlagerung von Acetylen an ungesättigte Ketone geprüft und die von M. F. Carroll erstmals beschriebene Kondensation $\alpha, \beta$-ungesättigter Alkohole mit Acetessigester auf verschiedene Beispiele übertragen. Das RaneyEisen erwies sich ferner als ein billiger und leicht zugänglicher Katalysator für die Hydrierung der Acetylen- zur Aethylenbindung. 